\title{
Characterization of Extracellular Dopamine Clearance in the Medial Prefrontal Cortex: Role of Monoamine Uptake and Monoamine Oxidase Inhibition
}

\author{
Hollie K. Wayment, ${ }^{1}$ James O. Schenk, ${ }^{1,2,3}$ and Barbara A. Sorg ${ }^{3,4}$ \\ Departments of ${ }^{1}$ Chemistry and ${ }^{2}$ Biochemistry and Biophysics, and ${ }^{3}$ Program in Neuroscience and ${ }^{4}$ Department of \\ Veterinary and Comparative Anatomy, Pharmacology and Physiology, Washington State University, Pullman, Washington \\ 99164
}

In vitro rotating disk electrode (RDE) voltammetry and in vivo microdialysis were used to characterize dopamine clearance in the rat medial prefrontal cortex (mPFC). RDE studies indicate that inhibition by cocaine, specific inhibitors of the dopamine transporter (DAT) and norepinephrine transporter (NET), and low $\mathrm{Na}^{+}$produced a $50-70 \%$ decrease in the velocity of dopamine clearance. Addition of the monoamine (MAO) inhibitors, L-deprenyl, clorgyline, pargyline, or in vivo nialamide produced $30-50 \%$ inhibition. Combined effects of uptake inhibitors with L-deprenyl on dopamine clearance were additive (up to 99\% inhibition), suggesting that at least two mechanisms may contribute to dopamine clearance. Dopamine measured extracellularly 5 min after exogenous dopamine addition to incubation mixtures revealed that most conditions of DAT/NET inhibition did not produce elevated dopamine levels above controls. Inhibition of MAO produced elevated dopamine levels only after long-term, but not short-term, incubation in vitro. Short-term incubation of L-deprenyl combined with DAT and NET uptake inhibitors increased dopamine above control levels, consistent with more than one mechanism of dopamine clearance. Local infusion of pargyline (100 or $300 \mu \mathrm{M})$ into the mPFC or striatum via microdialysis produced more pronounced and immediate increases in mPFC dopamine levels compared with striatum. Furthermore, dopamine elevation in the mPFC was not accompanied by a decrease in the dopamine metabolites, 3,4dihydroxyphenylacetic acid and homovanillic acid, as found in the striatum. These findings may have revealed a unique mechanism of mPFC dopamine clearance and therefore contribute to the understanding of multiple behaviors that involve mPFC dopamine transmission, such as schizophrenia, drug abuse, and working memory function.

Key words: dopamine; cocaine; dopamine transporter; medial prefrontal cortex; monoamine oxidase; rotating disk electrode voltammetry
Altered function of the medial prefrontal cortex (mPFC) has been implicated in multiple processes and behavioral disorders, including schizophrenia (Weinberger, 1995), drug abuse (Goeders and Smith, 1983; Isaac et al., 1989; Piazza et al., 1991; Schenk et al., 1991; Duvauchelle et al., 1992; McGregor and Roberts, 1995; Wolf et al., 1995; McGregor et al., 1996; Wise et al., 1996; Prasad et al., 1999), depression (Baxter et al., 1989; Tanda et al., 1994; Drevets, 1999; Juckel et al., 1999; Merriam et al., 1999; Rajkowska et al., 1999), and attention deficit hyperactivity disorder (Boix et al., 1998; Ernst et al., 1998; Puumala and Sirvio, 1998), as well as normal cognitive processes, including working memory function (Williams and Goldman-Rakic, 1995; Murphy et al., 1996; Cai and Arnsten, 1997; Jentsch et al., 1997a,b; Zahrt et al., 1997; Seamans et al., 1998; Wang, 1999) and decision making (Eslinger and Damasio, 1985; Damasio, 1995). Several of these studies have

\footnotetext{
Received April 5, 2000; revised Sept. 22, 2000; accepted Oct. 13, 2000.

This work was supported by Public Health Service Grants DA07384 (J.O.S.) and DA11787 (B.A.S). J.O.S. is also a recipient of an Independent Scientist Award (KO2 DA00184). We are grateful to Dr. Dale Edmondson (Emory University School of Medicine) for helpful discussions and to Dr. Weiran Wu and $\mathrm{Na} \mathrm{Li}$ for assistance with microdialysis studies.

Correspondence should be addressed to Dr. Barbara A. Sorg, Program in Neuroscience, Department of Veterinary and Comparative Anatomy, Pharmacology and Physiology, Washington State University, Pullman, WA 99164-6520. E-mail: barbsorg@vetmed.wsu.edu.

Dr. Wayment's present address: Ischemia Technologies, 6830 North Broadway, Suite A, Denver, CO 80142.

Copyright (C) 2001 Society for Neuroscience $\quad 0270-6474 / 01 / 210035-10 \$ 15.00 / 0$
}

focused in particular on altered dopaminergic functioning within the mPFC.

Despite the importance of prefrontal cortical dopamine in modulating cognition and behavior, little is known regarding processes that regulate extracellular clearance of dopamine in the mPFC. Inhibitors of dopamine transport demonstrate a weak effect on mPFC extracellular dopamine levels in vivo, including cocaine (Moghaddam and Bunney, 1989), amphetamine (Sorg et al., 1997; Pehek, 1999), nomifensine, and GBR 12909 (Cass and Gerhardt, 1995). The decreased responsiveness to dopamine uptake inhibitors may be explained partially by the lower terminal density and decreased number of dopamine transporters per terminal relative to striatal regions (Sesack et al., 1998). However, in vitro studies have shown that, in contrast to the striatum/ nucleus accumbens, dopamine uptake inhibitors only partially diminish dopamine uptake in the PFC (Hadfield and Nugent, 1983; Izenwasser et al., 1990; Elsworth et al., 1993; Wheeler et al., 1993). These findings suggest that an additional mechanism may contribute importantly to regulating clearance of extracellular dopamine in the mPFC.

Few studies have focused on measuring the kinetics of dopamine clearance in the mPFC. Garris et al. (1993) and Garris and Wightman (1994) have used in vivo voltammetry to examine clearance within the mPFC. Their findings suggest that dopamine clearance occurs over a large tissue volume because of the more restricted distribution of dopamine transporter (DAT) in this region, and they enhance the notion of volume transmission and 
the possible paracrine function of cortical dopamine. Cass and Gerhardt (1995) also have examined dopamine clearance in different regions of the mPFC using in vivo voltammetry. However, no studies to date have defined the contribution of metabolism to the kinetics of clearance, and some previous mPFC clearance studies are confounded by the factor of diff usion occurring in vivo. The present study used rotating disk electrode (RDE) voltammetry in vitro to characterize the kinetics of dopamine clearance in the $\mathrm{mPFC}$ and to determine regulatory processes that contribute to dopamine clearance. In vivo microdialysis in the $\mathrm{mPFC}$ and striatum was also performed to examine the effect of the monoamine (MAO) inhibitor, pargyline, on extracellular dopamine and metabolite levels.

\section{MATERIALS AND METHODS}

Animals and housing. Experiments were conducted according to the National Institutes of Health Guide for the Care and Use of Laboratory Animals, and experimental protocols were approved by the University animal care and use committee. Male Sprague Dawley rats weighing 250-300 gm were group-housed (three to four per cage) in a temperature- and humidity-controlled environment with ad libitum access to food and water. Animals were maintained on a $12 \mathrm{hr}$ light/dark schedule, with lights on at 7 A.M.

Drugs. Cocaine-hydrochloride was a gift from the National Institute on Drug Abuse. Dopamine, pargyline, clorgyline, desmethylimipramine (DMI), and nialamide were purchased from Sigma (St. Louis, MO), and GBR 12909, fluoxetine, and L-deprenyl were purchased from Research Biochemical Inc. (Natick, MA). All drugs used for RDE experiments were dissolved in distilled water and diluted to a final concentration in the incubation buffer. Solutions were made fresh each day and stored on ice.

$R D E$ voltammetry and HPLC. Unanesthetized rats were decapitated, and their brains were rapidly removed. The mPFC was dissected and weighed. The tissue was immediately chopped on an ice-cold glass plate and placed into $500 \mu \mathrm{l}$ of physiological buffer composed of (in $\mathrm{mm}$ ): $124 \mathrm{NaCl}, 1.80$ $\mathrm{KCl}, 1.30 \mathrm{MgSO}_{4}, 1.24 \mathrm{KH}_{2} \mathrm{PO}_{4}, 2.50 \mathrm{CaCl}_{2}, 26 \mathrm{NaHCO}_{3}, 10$ glucose, saturated with $95 \% \mathrm{O}_{2}-5 \% \mathrm{CO}_{2}$ gas mixture) as described (Meiergerd and Schenk, 1995; Earles et al., 1998; Earles and Schenk, 1999) and maintained at $37^{\circ} \mathrm{C}$ in a temperature-controlled chamber. Composition of the buffer in the low $\mathrm{Na}^{+}$condition consisted of replacement of $124 \mathrm{~mm} \mathrm{NaCl}$ with choline chloride. The tissue was disrupted by repetitive pipetting and washed by the repeated addition and removal of $250 \mu$ l fresh buffer seven times. The RDE (Pine Instruments, Grove City, PA) was lowered into the chamber and rotated at $2000 \mathrm{rpm}$, and a potential of $450 \mathrm{mV}$ relative to a $\mathrm{Ag} / \mathrm{AgCl}$ reference electrode was applied with a Bioanalytical Systems LC4B potentiostat (W. Lafayette, IN) with a $20 \mathrm{msec}$ time constant, defined as $5 \times$ resistance $\times$ capacitance. The tissue was incubated for 20 min until a stable baseline was reached. After the incubation period, dopamine was added to produce a final concentration of $2.0 \mu \mathrm{M}$, and its clearance was monitored on a Nicolet 310 digital oscilloscope. The concentration of dopamine used was based on a previous in vitro RDE study in the mPFC that demonstrated this concentration to be near $V_{\max }$ for dopamine clearance (Meiergerd et al., 1997). The initial rate of dopamine disappearance was estimated as described previously (Meiergerd and Schenk, 1995; Earles et al., 1998). The velocity of clearance was expressed as picomoles per second $\times$ grams of wet weight. This value was determined by calculating the slope of the line, as determined by time points taken every $20 \mathrm{msec}$, and converting from current to dopamine concentration by using a standard calibration curve prepared each day. The standard curve was generated using $0.1,0.25,0.5,1.0$, and $2.0 \mu \mathrm{M}$ dopamine. At a cumulative concentration of $1.85 \mu \mathrm{M}$ dopamine, clearance was followed for $25 \mathrm{sec}$ and is reported as the "buffer" sample throughout. The supernatant was prepared as described above for tissue preparation, but after washing seven times, the tissue was rapidly centrifuged and the supernatant removed for analysis of dopamine clearance, as described above. To determine dopamine clearance (in picomoles per second $\times$ grams of wet weight) for the buffer and supernatant, the mean tissue weight of all samples $(33.4 \mathrm{mg}$ ) was used.

The concentrations of DAT/norepinephrine transporter (NET) uptake inhibitors were chosen on the basis of the $K_{\mathrm{i}}$ or $\mathrm{IC}_{50}$ of each inhibitor and were $10-100$ times higher than the $K_{\mathrm{i}}$ or $\mathrm{IC}_{50}$ for each particular neurotransmitter. For DMI, both 0.1 and $100 \mu \mathrm{M}$ doses were used, with the former inhibiting only NET and the latter concentration inhibiting both
NET and DAT. A $0.1 \mu \mathrm{M}$ concentration of fluoxetine, which inhibits serotonin uptake, was chosen. Activity for NET may have been partially inhibited at this concentration of fluoxetine, the $K_{\mathrm{i}}$ of which has been reported as $\sim 0.2 \mu \mathrm{M}$ (Burke and Preskorn, 1995). For GBR 12909, a 3.0 $\mu \mathrm{M}$ solution was chosen, which is $\sim 50$ times higher than the $\mathrm{IC}_{50}$ for dopamine and approximately at the $K_{\mathrm{i}}$ for norepinephrine uptake (Heikkila and Manzino, 1984).

It should be noted that at the concentrations of L-deprenyl and clorgyline used $(100 \mu \mathrm{M})$, both MAO A and B would be expected to be inhibited. However, these agents as well as pargyline were examined because of previous work demonstrating an inhibitory effect of clorgyline (Lai et al., 1980; Fang and Yu, 1994) and L-deprenyl on dopamine uptake (Knoll, 1978; Lai et al., 1980; Zsilla et al., 1986; Knoll, 1992; Okuda et al., 1992; Fang and Yu, 1994), whereas pargyline has been reported not to influence dopamine uptake in the striatum (Fang and Yu, 1994).

After most treatments, at the end of a 5 min incubation, a $100 \mu \mathrm{l}$ sample was removed while the electrode was still rotating. Samples were placed into $20 \mu \mathrm{l}$ of $0.1 \mathrm{M}$ perchloric acid solution and immediately centrifuged to remove the tissue. The supernatant, which will be referred to as "aqueous phase" throughout, was collected and added to $50 \mu \mathrm{l}$ HPLC mobile phase containing $1 \times 10^{-7} \mathrm{M}$ isoproterenol and stored at $-80^{\circ} \mathrm{C}$ until assayed by HPLC for dopamine and its metabolites, 3,4dihydroxyphenylacetic acid (DOPAC), homovanillic acid (HVA), and 3-methoxytyramine (3-MT), as well as for NE. The results are reported as concentration of dopamine in micromolar taken from the original 100 $\mu$ sample removed at the end of the 5 min incubation. Determination of dopamine and metabolites, DOPAC, HVA, and 3-MT, and NE present in the aqueous phase was conducted by HPLC analyses according to Kalivas et al. (1988).

In vivo microdialysis. In vivo microdialysis was conducted in awake, unrestrained rats as described by Sorg et al. (1997). Animals were implanted with a chronic guide cannula into the $\mathrm{mPFC} \sim 1$ week before microdialysis experiments [for $\mathrm{mPFC}$ : anteroposterior $(\mathrm{AP})=+3.2 \mathrm{~mm}$ from bregma, mediolateral $(\mathrm{ML})=0.7 \mathrm{~mm}$, dorsoventral $(\mathrm{DV})=-1.5$ $\mathrm{mm}$ from skull; for striatum: $\mathrm{AP}=+1.0 \mathrm{~mm}$ from bregma, $\mathrm{ML}=2.2$ $\mathrm{mm}, \mathrm{DV}=-4.0 \mathrm{~mm}$ from skull, according to Paxinos and Watson (1998)]. Microdialysis probes with an active membrane region of $3 \mathrm{~mm}$ (mPFC) or $2 \mathrm{~mm}$ (striatum) were prepared as described (Sorg et al., 1997). Just before use, pargyline was diluted to its final concentration in artificial CSF (aCSF), which consisted of (in mM): 5.0 glucose, $5 \mathrm{KCl}, 120$ $\mathrm{NaCl}, 1.2 \mathrm{CaCl}_{2}, 1.2 \mathrm{MgCl}_{2}, 0.23$ sodium phosphate, $\mathrm{pH}$ 7.4. Probes were implanted the evening before the experiment, and on the next day, a minimum of 3-4 hr was allowed for a stable baseline to be obtained. After this, baseline samples were collected, and pargyline (either 100 or $300 \mu \mathrm{M}$ given to separate animals) was infused for a $60 \mathrm{~min}$ period and then replaced with aCSF for the remainder of the experiment. HPLC analyses of dopamine, DOPAC, and HVA were conducted as described by Sorg et al. (1997). Microdialysis probe placements within the mPFC and striatum were verified by cresyl violet staining of coronal brain sections (see Fig. 1, $B$ and $C$, respectively).

Data analyses and statistical testing. The percentage reduction in dopamine clearance was determined by subtracting the mean value for supernatant and buffer conditions, which were not significantly different from each other, from the values obtained for control and each treatment condition, dividing by the mean of the controls, and multiplying by 100 . The differences between means of the transport velocities (see Figs. 2-4), dopamine, its metabolites, and NE measured from sampled aqueous phase (see Fig. 5) were tested using a one-way ANOVA followed by a post hoc Fisher's test. Each neurotransmitter and metabolite was analyzed separately with an ANOVA (see Fig. 5). The microdialysis data (see Figs. 6, 7) were analyzed using a one-way repeated measures ANOVA followed by a Fisher's test to determine significant increases above the last baseline sample. All data were considered statistically significant at $p<0.05$.

\section{RESULTS}

Figure $1 A$ shows the brain area that was dissected for all experiments presented in Figures 2-5. This region included all mPFC regions anterior to $2.2 \mathrm{~mm}$ from bregma (Paxinos and Watson, 1998). Figure $1, B$ and $C$, shows photomicrographs of coronal brain sections demonstrating typical microdialysis probe placements in the mPFC and striatum for the data shown in Figures 6 and 7. In the mPFC, probes spanned the deeper layers of the cortex within the prelimbic and infralimbic cortices. 


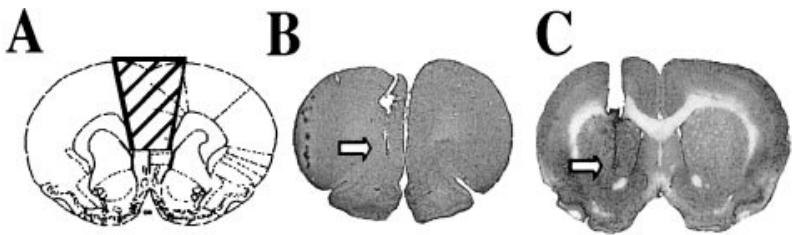

Figure 1. Coronal brain sections illustrating regions of the mPFC and striatum sampled in these studies. $A$, The portion of mPFC tissue removed for dopamine uptake studies. Shaded area represents the most caudal region used and includes all tissue rostral to the area shown. Diagram is taken from Paxinos and Watson (1998) at $+2.2 \mathrm{~mm}$ from bregma. $B$, Photomicrograph of a coronal brain section demonstrating microdialysis probe placement in the mPFC. The tip of the $3 \mathrm{~mm}$ probe is indicated by a white arrow. $C$, Photomicrograph of a coronal brain section demonstrating microdialysis probe placement in the striatum. The tip of the $2 \mathrm{~mm}$ probe is indicated by a white arrow.
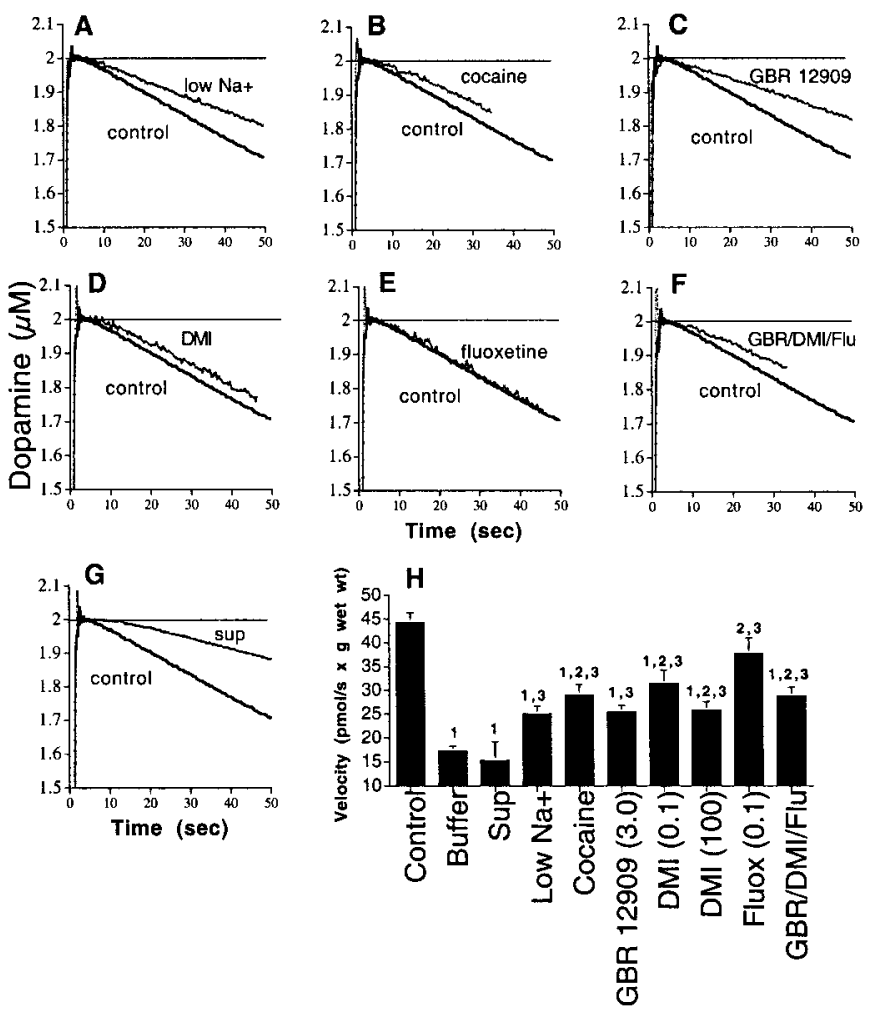

Figure 2. Velocity of $2.0 \mu \mathrm{M}$ dopamine clearance in $\mathrm{mPFC}$ tissue in the presence of low $\mathrm{Na}^{+}$or monoamine uptake inhibitors. $A-G$, Mean raw clearance profiles for dopamine. In some cases, velocity profiles were not collected from all animals for the entire period, and the lines are truncated $(B, D, F) . H$, Mean \pm SEM of dopamine clearance velocities shown in $A-G$. Mean values obtained in buffer and supernatant alone are shown for comparison. $n=16$ for control; $n=3-5$ for all other groups. ${ }^{1} p<0.05$, comparing with control condition; ${ }^{2} p<0.05$, comparing with buffer condition; ${ }^{3} p<0.05$, comparing with supernatant condition, as determined with a one-way ANOVA followed by a Fisher's test. Sup, Supernatant; Low Na ${ }^{+}, 26 \mathrm{mM} \mathrm{Na}^{+}$; Cocaine, $100 \mu \mathrm{M}$; GBR 12909, $3.0 \mu \mathrm{M}$; DMI, 0.1 or $100 \mu \mathrm{M}$ (summary bar graph only); Fluox, $0.1 \mu \mathrm{M}$ fluoxetine; $G B R / D M I / F l u$, combination of GBR $12909(3.0 \mu \mathrm{M})$, DMI $(0.1 \mu \mathrm{M})$, and fluoxetine $(0.1 \mu \mathrm{M})$.

\section{General characteristics of clearance of extracellular dopamine in the mPFC}

Figures 2-4 show graphs of the mean raw data taken from control and each of the various treatment groups presented along with a summary bar graph of the mean \pm SEM of dopamine clearance velocities. Clearance in the mPFC was found to be slower than that observed in tissues from the striatum or nucleus accumbens [compare with McElvain and Schenk (1992); Povlock and Schenk (1997), respectively] and generally consisted of a single linear phase. However, under some treatment conditions, two phases were observed and are described further in the results for Figure 4. For the summary bar graph presentation, all velocities that showed a single linear clearance phase were determined by calculating the slope from 5 to $25 \mathrm{sec}$, whereas those exhibiting biphasic profiles were calculated for the second phase (linear portion) from 20 to $30 \mathrm{sec}$. The slightly later time period of 20-30 sec was chosen for the graphs shown in Figure 4 because of the longer delay for the second phase of the velocity profile to appear. The buffer and supernatant conditions also exhibited biphasic profiles, and for these two conditions velocities were determined by calculating the slope from 15 to $25 \mathrm{sec}$.

The value of dopamine clearance in buffer (used in preparing the standard curve) was not significantly different from that observed in the supernatant preparation. By $30 \mathrm{sec}$ after dopamine addition to the incubation mixture containing the supernatant, the amount of dopamine cleared was $2.5 \%$ of the total dopamine added, and this disappearance may have been caused by the sum of oxidation of dopamine by the electrode, oxidation by dissolved $\mathrm{O}_{2}$, auto-oxidation of dopamine, or changes in the residual current of the electrode. The dopamine clearance profile for the buffer and supernatant are shown throughout Figures 2-4 for purposes of comparison.

\section{Monoamine uptake inhibitors}

Figure $2 A-G$ demonstrates the effect of low $\mathrm{Na}^{+}$and inhibitors of dopamine, NE, and serotonin (5-HT) uptake on the velocity of dopamine clearance in the mPFC. The data are presented in summary form (bar graph) in Figure $2 H$. The results indicate that $\sim 50-70 \%$ of the dopamine clearance rate is dependent on the function of the $\mathrm{Na}^{+} / \mathrm{Cl}^{-}$-dependent transporters DAT and NET. Low $\mathrm{Na}^{+}$buffer $(26 \mathrm{~mm} \mathrm{NaCl}$ vs $150 \mathrm{~mm}$ for the control condition) reduced dopamine velocity by $69 \%$. DMI added to a final concentration of $0.1 \mu \mathrm{M}$ to specifically inhibit NET reduced dopamine clearance velocity by $46 \%$, whereas a higher dose that inhibits both NET and DAT inhibited dopamine clearance velocity by $65 \%$. Although the higher concentration of DMI did not significantly alter dopamine clearance velocity as compared with the $0.1 \mu \mathrm{M}$ dose, the results suggest possible additive effects of uptake inhibitors acting at DAT and NET. The serotonin uptake inhibitor fluoxetine, given at $0.1 \mu \mathrm{M}$, did not significantly reduce the velocity of dopamine clearance. Addition of $3 \mu \mathrm{M}$ GBR 12909 , a DAT inhibitor, reduced dopamine clearance by $\sim 70 \%$. The effects of GBR 12909 and DMI were not additive, because the combination of the same concentrations of GBR 12909, DMI, and fluoxetine inhibited dopamine clearance velocity by only $55 \%$, and this combination was equally as effective as cocaine, which inhibits all three monoamine transporters. This may have been caused partially by the concentration of GBR 12909 used, which was close to the $\mathrm{IC}_{50}$ reported for norepinephrine in rat cortical tissue slices (Heikkila and Manzino, 1984).

\section{Monoamine oxidase inhibitors}

Figure $3 A-F$ shows the results of MAO inhibitors on the velocity of dopamine clearance in the $\mathrm{mPFC}$. The propargylamines, pargyline, clorgyline, and L-deprenyl, all reduced dopamine clearance velocity by $30-50 \%$. To determine whether a structurally different MAO inhibitor also attenuated the velocity of dopamine 


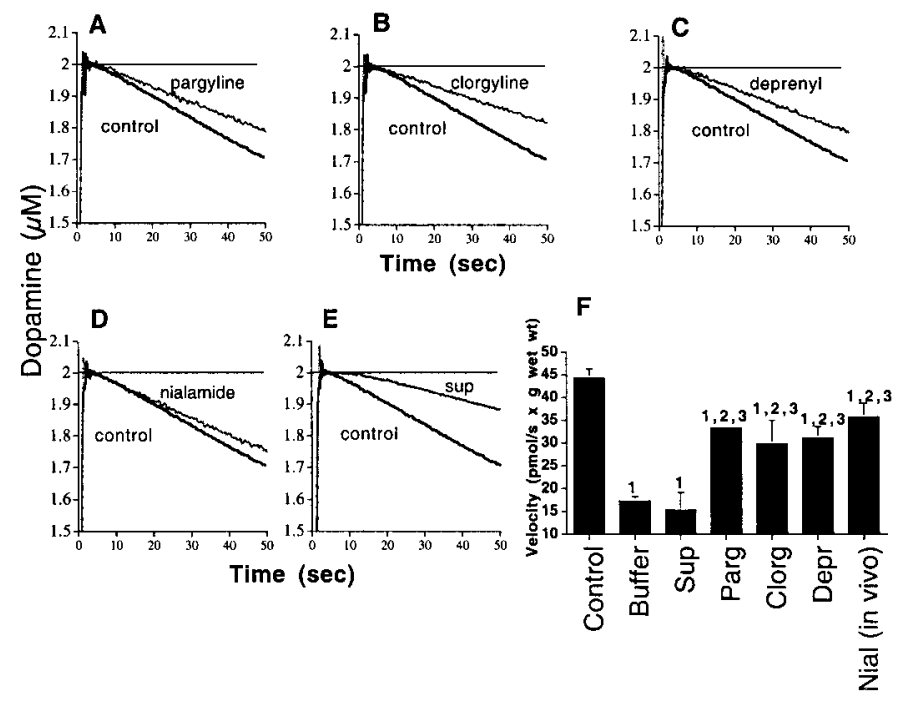

Figure 3. Velocity of $2.0 \mu \mathrm{M}$ dopamine clearance in mPFC tissue in the presence of MAO inhibitors. $A-E$, Mean raw clearance profiles for dopamine. $D$, Nialamide $(75 \mathrm{mg} / \mathrm{kg}$, i.p.) was administered in vivo $5 \mathrm{hr}$ before rats were killed. $F$, Mean \pm SEM of dopamine clearance velocities shown in $A-E$. Mean values obtained in buffer and supernatant alone are shown for comparison. $n=16$ for control; $n=3-5$ for all other groups. ${ }^{1} p<0.05$, comparing with control condition; ${ }^{2} p<0.05$, comparing with buffer condition; ${ }^{3} p<0.05$, comparing with supernatant condition, as determined with a one-way ANOVA followed by a Fisher's test. Sup, Supernatant; Parg, $100 \mu \mathrm{M}$ pargyline; Clorg, $100 \mu \mathrm{M}$ clorgyline; Depr, 100 $\mu \mathrm{M}$ deprenyl; Nial, $75 \mathrm{mg} / \mathrm{kg}$ (i.p.) nialamide in vivo.

clearance, nialamide was administered to rats systemically. Nialamide was administered systemically because previous in vitro experiments using RDE demonstrated that nialamide nonspecifically alters electrode responses (J. O. Schenk, unpublished observations). This dose of nialamide $(75 \mathrm{mg} / \mathrm{kg}$, i.p.) $5 \mathrm{hr}$ before rats were killed (Hovevey-Sion et al., 1989) resulted in a $\sim 30 \%$ inhibition of dopamine clearance rate.

\section{Inhibition of DAT/NET uptake and MAO activity}

The time-dependent effects of DAT/NET and MAO inhibition were examined by adding these inhibitors simultaneously with dopamine to the incubation media $(t=0 \mathrm{sec})$. For Figure 4 , all inhibitor additions to the incubation were given simultaneously with exogenous dopamine addition at $t=0 \mathrm{sec}$. Figure $4 A-F$ shows that a biphasic clearance profile was observed in each case when these inhibitors were added at $t=0 \mathrm{sec}$. The bar graph in Figure $4 G$ summarizes the means \pm SEM of dopamine clearance occurring from 20 to $30 \mathrm{sec}$. The early component of the biphasic response demonstrated inhibition of dopamine clearance that lasted for several seconds. This initial inhibition lasted significantly longer under conditions in which cocaine + L-deprenyl were added compared with all other conditions.

Figure $4 A$ demonstrates that inhibition by cocaine added at $t=$ $0 \mathrm{sec}$ was greater than that observed for cocaine when it was added $30 \mathrm{sec}$ before dopamine addition, although these values were not significantly different from each other ( $p=0.23)$ (compare with Fig. $2 B, H$ ). There was a trend for cocaine given at $t=$ $0 \mathrm{sec}$ to cause a lower rate of dopamine clearance than the buffer and supernatant conditions, but the value from cocaine at $t=0$ sec shown in the summary bar graph was not statistically different from those of the buffer and supernatant conditions (Fig. 4G). $\mathrm{L}$-deprenyl addition to the incubation at $t=0 \mathrm{sec}$ also resulted in a biphasic profile and only partially inhibited the clearance veloc-
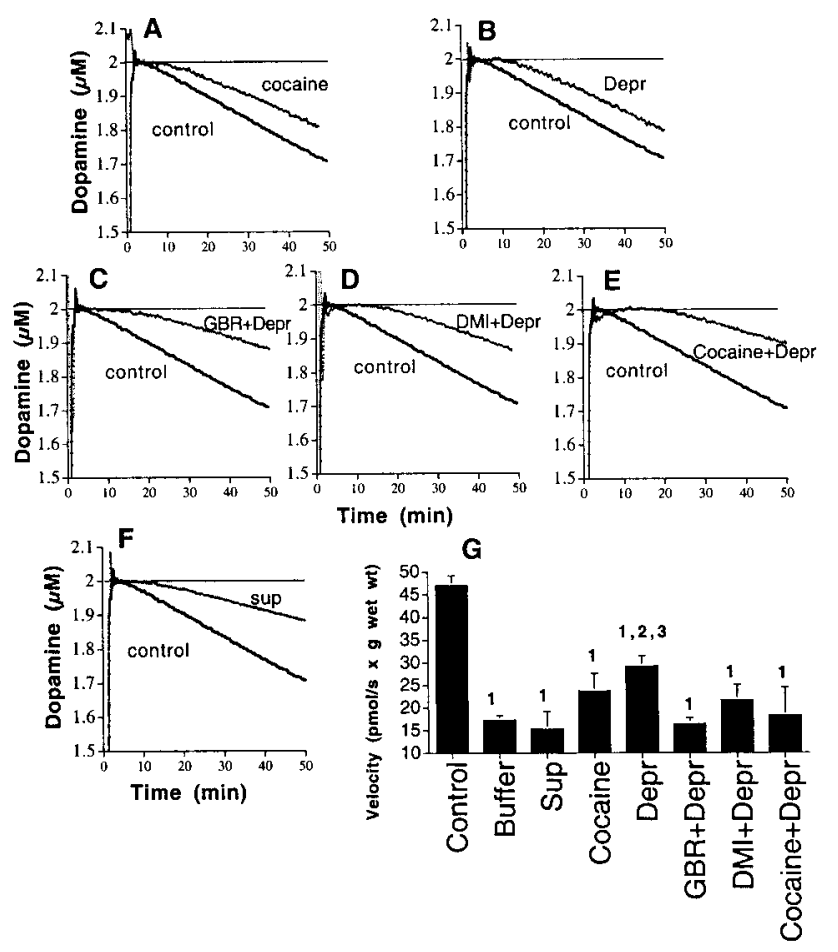

Figure 4. Velocity of $2.0 \mu \mathrm{M}$ dopamine clearance in mPFC tissue in the presence of cocaine, L-deprenyl, or the combination of L-deprenyl with GBR $12909(3.0 \mu \mathrm{M})$, DMI $(0.1 \mu \mathrm{M})$, or cocaine $(100 \mu \mathrm{M}) . A-C$, Mean raw clearance profiles for dopamine. For $A-E$, all components were added simultaneously with $2.0 \mu \mathrm{M}$ dopamine. $G$, Mean \pm SEM of dopamine clearance velocities shown in $A-F$. Mean values obtained in buffer and supernatant alone are shown for comparison. $n=16$ for control; $n=3-5$ for all other groups. ${ }^{1} p<0.05$, comparing with control condition; ${ }^{2} p<0.05$, comparing with buffer condition; ${ }^{3} p<0.05$, comparing with supernatant condition, as determined with a one-way ANOVA followed by a Fisher's test. Sup, Supernatant; Cocaine, $100 \mu \mathrm{M}$; Depr, $0.1 \mu \mathrm{M}$ deprenyl; $G B R+D e p r, D M I+D e p r$, and Cocaine + Depr were added to the incubation in the same concentrations as used singly.

ity of dopamine. This value, shown in the summary bar graph, was significantly reduced from control, although significantly higher than the values for the buffer and supernatant conditions. Inhibition by L-deprenyl added at $t=0 \mathrm{sec}$ was not different from that produced by L-deprenyl present in the incubation mixture for 20 min before dopamine addition $(p=0.61)$ (compare with Fig. $3 C, F)$.

Cocaine and other DAT and NET inhibitors were combined with the MAO inhibitor L-deprenyl to determine whether the partial inhibitory effects on dopamine clearance velocity described above were additive when assessed over the 20-30 sec range. Figure $4 C-E, G$ demonstrates that additive effects of L-deprenyl occurred when combined with these uptake inhibitors, with percentage inhibition ranging from 83\% (DMI + L-deprenyl) to $99 \%$ (GBR + L-deprenyl), with a $94 \%$ inhibition by cocaine + L-deprenyl (refer to summary bar graph in Fig. $2 \mathrm{H}$ for effects of DMI and GBR 12909 given alone).

\section{Dopamine metabolism}

For most of the RDE experiments, samples of aqueous phase from brain homogenates were collected $5 \mathrm{~min}$ after the addition of dopamine to determine the levels of dopamine and its metabolites, DOPAC, HVA, and 3-MT. Norepinephrine was also measured, because dopamine is taken up into NE terminals via NET. The relatively longer time point of $5 \mathrm{~min}$ was chosen to increase 


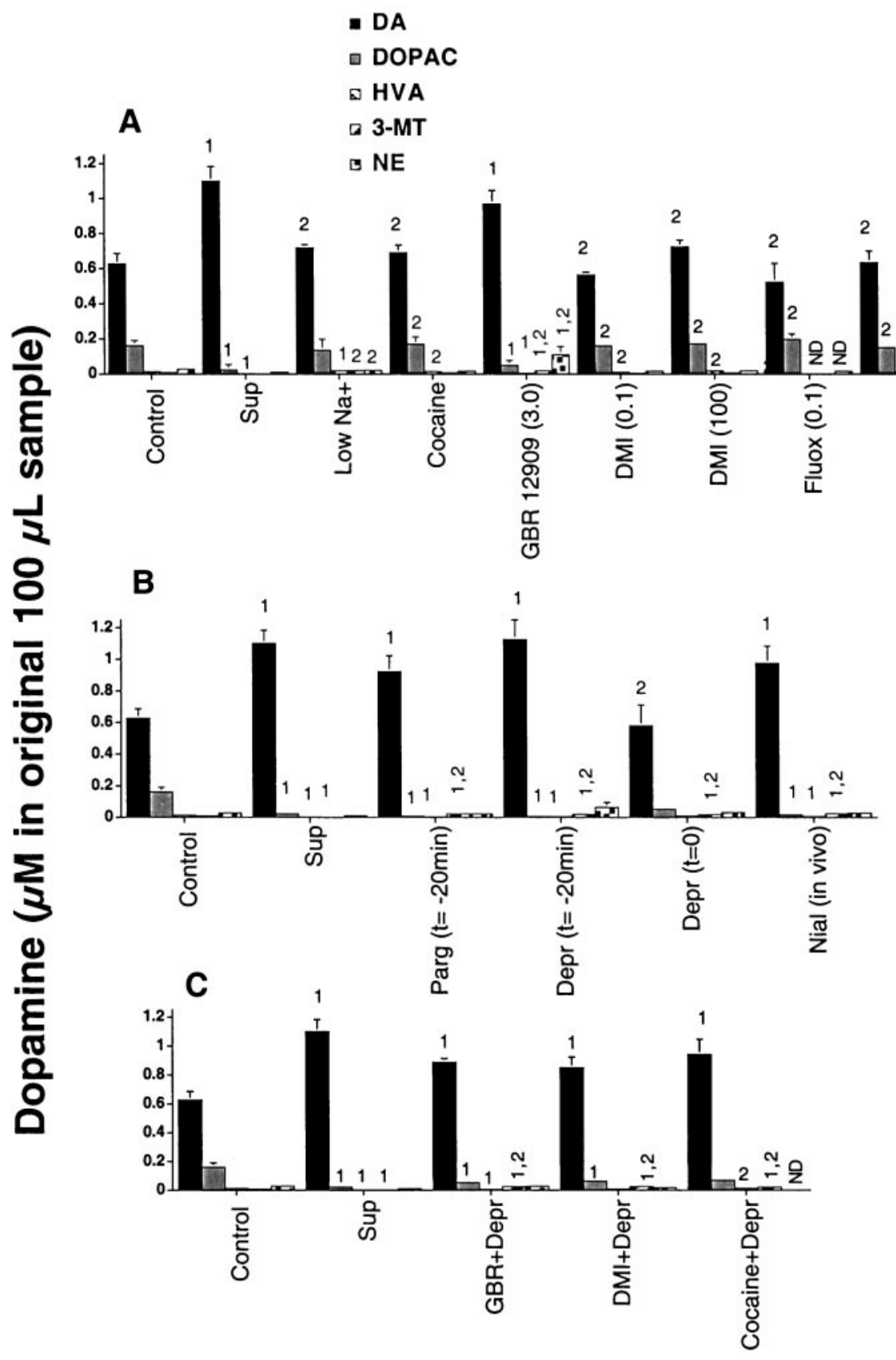

Figure 5. Concentration of dopamine, metabolites, and NE in the aqueous phase from mPFC tissue 5 min after incubation in the presence of dopamine under low $\mathrm{Na}^{+}$conditions or after addition of monoamine uptake/MAO inhibitors. Data represent mean \pm SEM of dopamine, DOPAC, HVA, 3-MT, and NE concentrations from the original $(100 \mu \mathrm{l})$ sample taken from the incubation. $A$, Monoamine uptake inhibitors $(n=15$ for control; $n=3-5$ for all other groups). $B$, MAO inhibitors ( $n=15$ for control; $n=3-4$ for all other groups). $C$, Combination of L-deprenyl with uptake inhibitors GBR 12909 (GBR+Depr), DMI (DMI+Depr), or cocaine (Cocaine + Depr) $(n=15$ for control; $n=$ 3-5 for all other groups). ${ }^{1} p<0.05$, comparing with control condition; ${ }^{2} p<0.05$, comparing with supernatant condition, as determined with a oneway ANOVA followed by a Fisher's test. $N D$, Not determined. the possibility that metabolic pathways of dopamine may be traced after the various treatment conditions. Before the addition of $2.0 \mu \mathrm{M}$ dopamine, basal concentrations from separate experiments were (in micromolar per original sample removed) as follows: dopamine, $0.0048 \pm 0.0003$; DOPAC, $0.0085 \pm 0.0012$; HVA, $0.0102 \pm 0.0026$; 3-MT, $0.0328 \pm 0.0078$; NE, $0.0058 \pm$ $0.0008(n=4)$.

Figure $5 A$ corresponds to the aqueous phase taken from experiments shown in Figure $2 A-G$ in which dopamine uptake inhibitors were added to the incubation medium. Five minutes after the addition of dopamine, the level of dopamine on the outside (aqueous phase) of controls was $0.627 \mu \mathrm{M}$. The level of dopamine remaining in the aqueous phase of the supernatant condition was significantly increased, whereas the metabolites, DOPAC and HVA, were significantly decreased compared with the control condition. Dopamine, metabolites, and NE values for all condi- tions were compared with both the control and supernatant conditions, and significant differences are indicated in Figure 5. However, for purposes of clarity, comparison of these values with those from the supernatant will be discussed here only for dopamine, because several metabolites were significantly higher when the levels from tissue were compared with those from the supernatant condition.

The only uptake inhibitor that produced a significant increase in aqueous phase dopamine levels above control values after the 5 min incubation was GBR 12909, whereas no other conditions of dopamine, NE, or 5-HT uptake inhibition produced an increase in aqueous phase dopamine levels. The combination of GBR 12909 with DMI or fluoxetine also did not produce a significant elevation in aqueous phase dopamine levels. The levels of DOPAC and HVA measured in the aqueous phase after the 5 min incubation were significantly decreased with GBR 12909 
addition, whereas 3-MT and NE levels were significantly elevated after GBR 12909 addition. The only other significant change from the control condition was an increase in HVA levels in the low $\mathrm{Na}^{+}$condition.

Figure $5 B$ shows dopamine, its metabolites, and NE levels in the aqueous phase after the addition of MAO inhibitors corresponding to experiments shown in Figures $3 A, C-E$ and $4 B$. Dopamine levels were significantly elevated after all conditions of MAO inhibition, with the exception of the condition in which L-deprenyl was added at $t=0 \mathrm{sec}$ and incubated for the $5 \mathrm{~min}$ period. In all conditions, with the exception of L-deprenyl added simultaneously with dopamine $(t=0 \mathrm{sec})$, the MAO inhibitors significantly reduced DOPAC levels. L-deprenyl added at $t=0$ min attenuated, but did not significantly reduce, DOPAC levels. Homovanillic acid levels were also significantly reduced after pargyline and L-deprenyl addition when these drugs were present during the $20 \mathrm{~min}$ baseline stabilization period. The levels of 3-MT in the supernatant were elevated above control values under all conditions of MAO inhibition. Norepinephrine levels were not significantly altered by any of the MAO inhibitors.

Figure $5 C$, corresponding to the aqueous phase taken from experiments shown in Figure $4 C-F$, demonstrates that the combination of GBR $12909+$ L-deprenyl, DMI + L-deprenyl, and cocaine + L-deprenyl all significantly increased dopamine levels above control values and were not different from levels in the supernatant condition. The levels of DOPAC were significantly decreased from controls after the combination of L-deprenyl with either GBR 12909 or DMI. The combination of L-deprenyl with GBR 12909 also significantly decreased HVA levels. As with the MAO inhibitors alone, 3-MT levels were also increased when given in combination with these uptake inhibitors. Norepinephrine levels were not determined for the cocaine + L-deprenyl group, but no other treatment conditions produced significant differences in this neurotransmitter.

\section{Microdialysis}

In vivo microdialysis experiments were conducted in the mPFC and striatum to determine whether (1) local inf usion of the MAO inhibitor, pargyline, would produce an increase in extracellular dopamine levels and (2) whether there were differential effects between these two brain areas in the regulation of dopamine and metabolites by local pargyline infusion. The results of this experiment are shown in Figures $6 A-F(100 \mu \mathrm{M}$ pargyline) and $7 A-F$ (300 $\mu \mathrm{M}$ pargyline). Infusion with $100 \mu \mathrm{M}$ pargyline produced a significant elevation in extracellular dopamine levels in the mPFC when infused at a concentration of $100 \mu \mathrm{M}$ for a $1 \mathrm{hr}$ period. This increase was $\sim 300 \%$ above baseline values and occurred within 20-40 min after pargyline infusion. A significant increase in DOPAC levels was found, whereas no significant changes occurred for HVA levels. In contrast, striatal dopamine levels showed only a trend toward an increase to $\sim 200 \%$ above baseline values, but the trend toward an increase above baseline was delayed by $20 \mathrm{~min}$ and occurred only after significant reduction in DOPAC levels, which were reduced to $\sim 50 \%$ of baseline. Also in contrast to the mPFC, HVA levels in the striatum were significantly reduced to $\sim 40 \%$ of baseline.

In the presence of $300 \mu \mathrm{M}$ pargyline, dopamine levels in the mPFC increased by $\sim 5000 \%$ above baseline values, and this effect occurred within 20-40 min after pargyline infusion. Again, no significant decrease in DOPAC or HVA levels was observed. Striatal dopamine increased to $\sim 1000 \%$ above baseline levels, but as with the lower dose of pargyline, the effects were delayed by 20

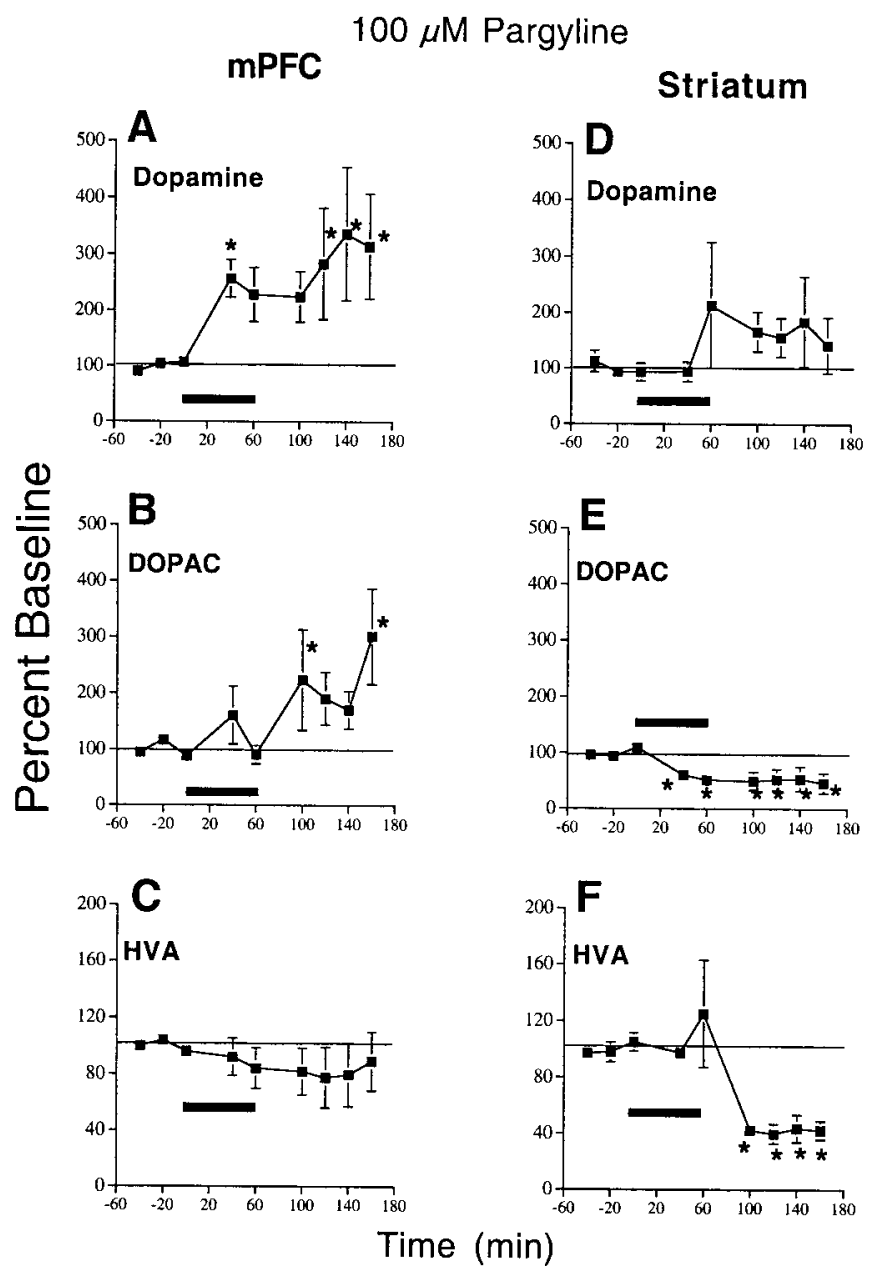

Figure 6. In vivo microdialysis measurements of extracellular dopamine, DOPAC, and HVA levels in the mPFC and striatum after $100 \mu \mathrm{M}$ pargyline infusion. Data represent mean \pm SEM of dopamine, DOPAC, and HVA levels before pargyline inf usion, during a $1 \mathrm{hr}$ infusion with 100 $\mu \mathrm{M}$ pargyline in the mPFC $(A-C)$ or striatum $(D-F)$. Pargyline inf usion is indicated by the black bar and was then replaced with aCSF. Samples collected for the first time point after manual switching of solutions is not shown because of alterations in aCSF flow rates. Basal levels from mPFC from all groups (including those shown in Fig. 7) were (in femtomoles per sample) as follows: for dopamine, $13.0 \pm 2.4$; for DOPAC, $247 \pm 58$; for HVA, $404 \pm 57$. Basal levels from striatum from all groups (including those shown in Fig. 7) were (in femtomoles per sample) as follows: for dopamine, $48.8 \pm 9.9$; for DOPAC, $7219 \pm 1286$; for HVA, $6087 \pm 1009$. Sample sizes were as follows: mPFC dopamine, $n=9$; DOPAC and HVA, $n=7$; striatal dopamine, DOPAC, and HVA, $n=6 .{ }^{*} p<0.05$ compared with last baseline sample, as determined with a one-way, repeatedmeasures ANOVA followed by a Fisher's test.

min as compared with the early increase in mPFC dopamine levels. The levels of DOPAC, although reduced to $\sim 40 \%$ of baseline, did not reach statistical significance because all values were compared with the last baseline sample, which was already decreased by $20 \%$ before pargyline infusion. HVA levels were significantly reduced to $30 \%$ of baseline levels.

\section{DISCUSSION}

The main findings from these studies are as follows. (1) DAT and NET inhibitors account for only $50-70 \%$ of the velocity of dopamine clearance in the mPFC; (2) MAO inhibitors attenuate the velocity of dopamine clearance by $\sim 30-50 \%$; (3) the effects of DAT/NET uptake inhibitors plus the MAO inhibitor, L-deprenyl, 


\section{$300 \mu \mathrm{M}$ Pargyline}
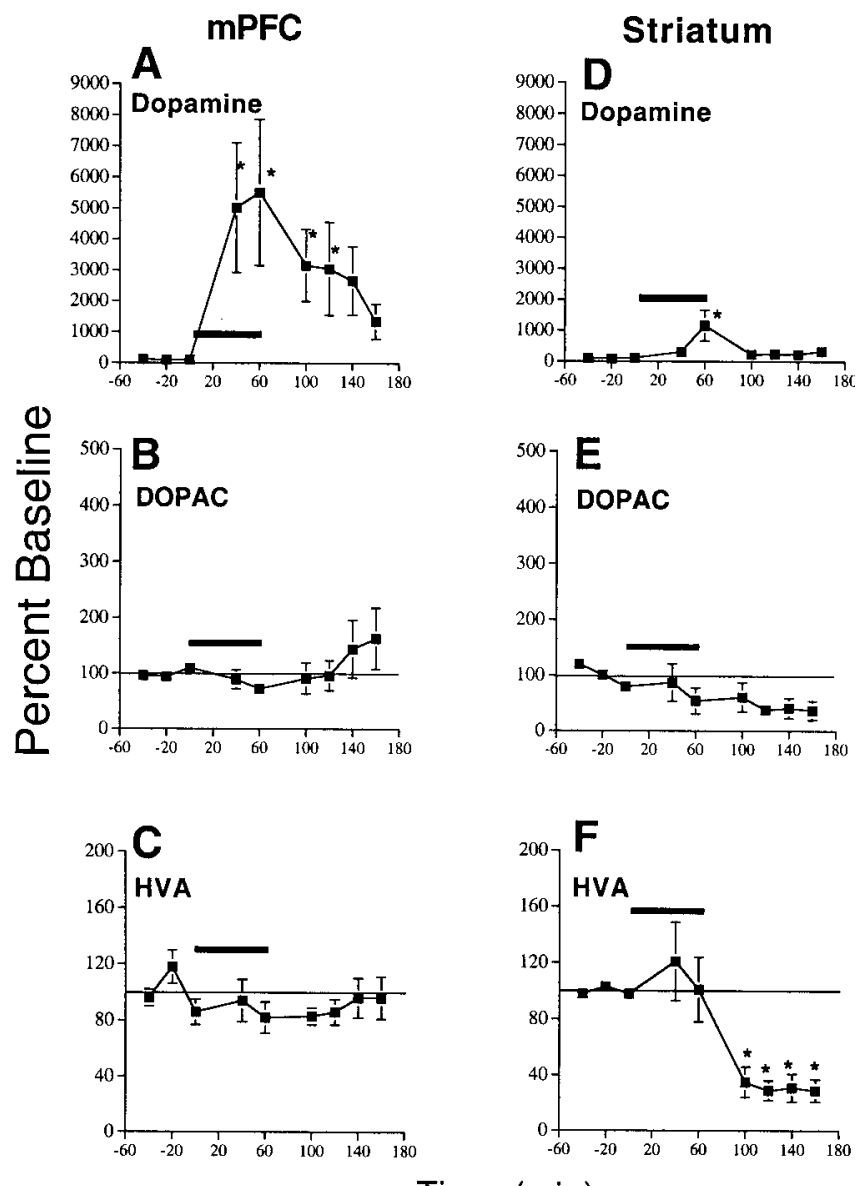

Time $(\min )$

Figure 7. In vivo microdialysis measuring extracellular dopamine, DOPAC, and HVA levels in the mPFC and striatum after $300 \mu \mathrm{M}$ pargyline infusion. Data represent mean \pm SEM of dopamine, DOPAC, and HVA levels before pargyline inf usion, during a $1 \mathrm{hr}$ inf usion with 300 $\mu \mathrm{M}$ pargyline in the mPFC $(A-C)$ or striatum $(D-F)$. Pargyline infusion is indicated by the black bar and was then replaced with aCSF. Samples collected for the first time point after manual switching of solutions are not shown because of alterations in aCSF flow rates. Sample sizes were as follows: mPFC dopamine and DOPAC, $n=9$; HVA, $n=8$; striatal dopamine, DOPAC, and HVA, $n=5$. ${ }^{*} p<0.05$ compared with last baseline sample, as determined with a one-way, repeated-measures ANOVA followed by a Fisher's test.

on dopamine clearance appear to be additive; (4) the effect of DAT/NET inhibition on the initial rate of dopamine clearance may be compensated for by another process; and (5) local in vivo pargyline infusion into the $\mathrm{mPFC}$ via microdialysis dramatically elevates mPFC extracellular dopamine levels with no decreases in DOPAC or HVA, whereas pargyline-induced dopamine increases in the striatum occur only after infusion of the higher pargyline dose and are accompanied by decreases in DOPAC or HVA levels, or both.

\section{Processes of mPFC dopamine clearance in vitro}

The RDE findings are most consistent with the possibility that there are at least two major processes by which dopamine is cleared from the extracellular space. One is by a $\mathrm{Na}^{+}$-dependent process, presumably via DAT and NET, and the second is by a process altered by MAO inhibitors. Several previous in vitro studies have also reported only a partial $(\sim 40-70 \%)$ inhibition of dopamine clearance in the mPFC after cocaine or low $\mathrm{Na}^{+}$ conditions, or both, in contrast to the nucleus accumbens and striatum, in which cocaine inhibits dopamine clearance by $95 \%$ (Hadfield and Nugent, 1983; Izenwasser et al., 1990; Elsworth et al., 1993; Wheeler et al., 1993).

The time course of inhibition by L-deprenyl on dopamine clearance velocity suggests that there is an immediate effect of MAO inhibitors on dopamine clearance. Several possibilities for this response are considered.

One explanation for the effect of L-deprenyl is that deprenyl is converted to L-amphetamine, and this in turn inhibits dopamine uptake via DAT or NET (Karoum et al., 1982; Tetrud and Langston, 1989; Okudo et al., 1992). However, all MAO inhibitors tested in the present study decreased the dopamine clearance rate. Therefore, there may be an alternative process in the mPFC that is inhibited by all of these agents.

A second possibility is that MAO inhibitors alter the quinpirole binding site, which in turn may decrease dopamine clearance velocity. MAO inhibitors have been shown to modulate the binding of quinpirole (Levant et al., 1993, 1996). If the quinpirole binding site on D2 or D3 receptors in the mPFC is bound by MAO, modification of DAT function might be expected, given that D2 receptors have been shown to regulate DAT activity (Meiergerd et al., 1993; Cass and Gerhardt, 1994; Batchelor and Schenk, 1998). However, such an explanation is not consistent with the partial effects of low $\mathrm{Na}^{+}$or DAT/NET uptake inhibitors.

A third explanation is that these MAO inhibitors may inhibit other more recently described transporter systems, organic cation transporter (OCT) 2 or OCT3 (Busch et al., 1998; Wu et al., 1998; Grundemann et al., 1999). Although both of these transporters have been reported to transport dopamine and are present in the brain (Gorboulev et al., 1997), the OCT3 transporter appears much more abundant in brain tissue than OCT2 and is found in cortical regions (Wu et al., 1998). The OCT3 transporter mediates the uptake of dopamine, and amphetamine interacts with this transporter as well (Wu et al., 1998). Thus, interaction with this newly described transporter may be important in mPFC dopamine clearance.

Finally, there is the possibility that different clearance processes are present within different heterogenous regions of the mPFC. Previous work has demonstrated regional effects of DAT and NET inhibitors (Cass and Gerhardt, 1995), and regional differences in dopamine clearance may be expected based on immunohistochemical measures of DAT location (Ciliax et al., 1995). Our studies examined the entire mPFC and therefore would not distinguish among clearance processes located within different mPFC subregions.

\section{Biphasic effects of cocaine and MAO inhibition on dopamine clearance}

Unexpectedly, when either cocaine or the MAO inhibitor, L-deprenyl, was added simultaneously with dopamine to the in vitro incubation mixture, there was a biphasic profile of dopamine clearance rather than a linear clearance profile. The first portion lasted on the order of seconds and was nearly or completely blocked by these agents for several seconds. The reason for observing complete inhibition of dopamine clearance for a longer period than what was observed in the supernatant condition is not clear. It should be pointed out that no alterations in baseline output were observed after cocaine or pargyline were added to mPFC tissue in the absence or presence of exogenous dopamine addition, nor did 
these drugs cause release of dopamine from mPFC tissue. In addition, neither pargyline nor cocaine alters sensitivity of the electrode to dopamine (data not shown). Future studies will need to directly address the biphasic nature of dopamine clearance in this brain region.

The second phase of the biphasic profile demonstrated an additive effect for L-deprenyl with uptake inhibitors, suggesting that a second process or multiple processes may work either separately or in tandem with DAT and NET function.

\section{Dopamine and metabolites in vitro}

The results from the HPLC analyses of aqueous phase samples taken 5 min after exogenous dopamine addition indicated that dopamine levels were significantly elevated only after GBR 12909 addition but, unexpectedly, not in the presence of cocaine or the combination of GBR 12909, DMI, and fluoxetine. It is unclear why GBR 12909 alone would produce greater effects than with the combination of DAT/NET inhibitors. The results suggest that GBR 12909 may have alternative actions, such as direct inhibition of the MAO inhibitor-dependent process, or that binding of cocaine or DMI to NET may activate a process that is blocked by MAO inhibitors. Any effects of MAO inhibitors on dopamine clearance dependent on NET activity would not be expected to occur in brain areas that lack substantial clearance by NET, such as the nucleus accumbens or striatum. Consistent with the absence of effects of MAO inhibitors on dopamine clearance in these latter brain regions, pargyline addition does not alter dopamine clearance velocity in the nucleus accumbens or striatum when tested in the RDE system that has been used in the present studies (Meiergerd and Schenk, 1994; Povlock and Schenk, 1997).

Dopamine was elevated in the aqueous phase after all conditions of MAO inhibition, with the exception of when L-deprenyl was added at $t=0 \mathrm{sec}$. L-deprenyl incubated over a short-term period (added at $t=0 \mathrm{sec}$ ) produced immediate partial inhibitory effects on dopamine clearance yet did not alter extracellular dopamine levels after the 5 min incubation period, perhaps because of compensatory DAT and NET activity. However, over the longer incubation time (20 min or after in vivo administration), MAO inhibitors, including L-deprenyl, produced elevated dopamine levels in the aqueous phase, suggesting the possibility that uptake by DAT and NET may be impaired after longer incubation with MAO inhibitors. When L-deprenyl ( $t=0 \mathrm{sec})$ was combined with DAT/NET uptake inhibitors, extracellular dopamine levels were significantly elevated above controls. Together, these findings support the results from the RDE studies suggesting that at least two processes contribute importantly to dopamine clearance in the mPFC.

\section{Pargyline effects on MPFC and striatal extracellular dopamine levels in vivo}

Microdialysis studies examining MAO inhibitor action on extracellular dopamine levels have used systemic injection of these drugs, with either increases (Sharp et al., 1986; Butcher et al., 1990; Okudo et al., 1992) or no changes reported (Kato et al., 1986; Butcher et al., 1990). In the present study, pargyline concentrations prepared for inf usion through the microdialysis probe were higher than those shown to inhibit MAO activity (Cesura and Pletscher, 1992). However, it was not possible to know the concentration of parygline reaching the surrounding tissue, and substantial increases in extracellular dopamine levels occurred in the mPFC in the absence of decreases in the levels of its metabolites, DOPAC and HVA, indicating an effect of pargyline at least partially independent of its MAO inhibitory action. In contrast, striatal dopamine levels were elevated by pargyline infusion to a lesser degree and were accompanied by decreases in DOPAC and HVA levels. Together, these data suggest that mPFC dopamine is regulated differently from striatal dopamine and may be caused by a direct effect of pargyline on mPFC dopamine uptake processes, although contribution by long-loop feedback pathways cannot be ruled out. Future in vivo studies should examine whether additive effects occur for mPFC dopamine using lower concentrations of MAO inhibition combined with DAT/NET uptake inhibition.

In summary, these results demonstrate that more than one major mechanism appears responsible for dopamine clearance in the mPFC: (1) clearance occurs by DAT and NET, and (2) clearance occurs by a second component that is blocked by MAO inhibitors. The kinetics of dopamine clearance in vitro suggest that the effects of DAT and NET inhibitors combined with the MAO inhibitor, L-deprenyl, are additive. This is the first report describing an important contribution by MAO inhibitors for the clearance of $\mathrm{mPFC}$ dopamine in the kinetic domain. In vivo microdialysis studies infusing the MAO inhibitor, pargyline, into the $\mathrm{mPFC}$ suggest that pronounced increases in extracellular dopamine levels occur in the absence of decreases in extracellular DOPAC or HVA levels, whereas pargyline infusion into the striatum produces less pronounced increases that appear to be a consequence of decreased dopamine metabolism. Such findings may have implications for reinterpreting the role of MAO inhibitors in antidepressant action and offer caution against extrapolation of observations across different brain regions when examining MAO inhibitor action and monoamine transporter function. The ability to uniquely control extracellular dopamine levels within the mPFC may have importance for several psychopathological behaviors, including schizophrenia and drug abuse, as well as normal cognitive processes such as working memory.

\section{REFERENCES}

Batchelor M, Schenk JO (1998) Protein kinase A kinetically upregulates the striatal transporter for dopamine. J Neurosci 18:10304-10309.

Baxter L, Schwartz JM, Phelps E, Mazziotta JC, Guze BH, Selin CE, Gerner RH, Sumida RM (1989) Reduction of prefrontal glucose metabolism common to three types of depression. Arch Gen Psychiatry 46:243-250.

Boix F, Qiao SW, Kolpus T, Sagvolden T (1998) Chronic L-deprenyl treatment alters brain monoamine levels and reduces impulsiveness in an animal model of attention-deficit/hyperactivity disorder. Behav Brain Res 94:153-162.

Burke MJ, Preskorn SH (1995) Short-term treatment of mood disorders with standard antidepressants. In: Psychopharmacology: the fourth generation of progress (Bloom FE, Kupfer DJ, eds), pp 1053-1079. New York: Raven.

Busch AE, Karbach U, Miska D, Gorboulev V, Akhoundova A, Volk C, Arndt P, Ulzheimer JC, Sonders MS, Baumann C, Waldegger S, Lang F, Koepsell H (1998) Human neurons express the polyspecific cation transporter hOCT2, which translocates monoamine neurotransmitters, amantadine, and memantine. Mol Pharmacol 54:342-352.

Butcher SP, Fairbrother JS, Kelly JS, Arbuthnott GW (1990) Effect of selective monoamine oxidase inhibitors on the in vivo release and metabolism of dopamine in the rat striatum. J Neurochem 55:981-988.

Cai JX, Arnsten AFT (1997) Dose-dependent effects of the dopamine D1 receptor agonists A77636 or SKF81297 on spatial working memory in aged monkey. J Pharmacol Exp Ther 283:183-189.

Cass WA, Gerhardt GA (1994) Direct in vivo evidence that D2 dopamine receptors can modulate dopamine uptake. Neurosci Lett 176:259-263.

Cass WA, Gerhardt GA (1995) In vivo assessment of dopamine uptake in rat medial prefrontal cortex: comparison with dorsal striatum and nucleus accumbens. J Neurochem 65:201-207.

Cesura AM, Pletscher A (1992) The new generation of monoamine oxidase inhibitors. In: Progress in drug research, Vol 38 (Jucker E, ed), pp 171-298. Boston: Birhauser Verlag.

Ciliax BJ, Heilman C, Demchyshyn LL, Pristupa ZB, Ince E, Hersch SM, 
Niznik HB, Levey AI (1995) The dopamine transporter: immunochemical characterization and localization in brain. J Neurosci $15: 1714-1723$

Damasio AR (1995) On some functions of the human prefrontal cortex. Ann NY Acad Sci 769:241-251.

Drevets WC (1999) Prefrontal cortical-amygdalar metabolism in major depression. Ann NY Acad Sci 877:614-637.

Duvauchelle CL, Levitin M, MacConell LA, Lee LK, Ettenberg A (1992) Opposite effects of prefrontal cortex and nucleus accumbens infusions of flupenthixol on stimulant-induced locomotion and brain stimulation reward. Brain Res 576:104-110.

Earles C, Schenk JO (1999) Rotating disk electrode voltammetric measurements of dopamine transporter activity: an analytical evaluation. Anal Biochem 264:191-198.

Earles C, Wayment HK, Green M, Schenk JO (1998) Resolution of biogenic amine transporter kinetics by rotating disk electrode voltammetry: methodology and mechanistic interpretations. Methods Enzymol 296:660-675.

Elsworth JD, Taylor JR, Berger P, Roth RH (1993) Cocaine-sensitive and -insensitive dopamine uptake in prefrontal cortex, nucleus accumbens and striatum. Neurochem Int 23:61-69.

Ernst M, Zametkin AJ, Matochik JA, Jons PH, Cohen RM (1998) DOPA decarboxylase activity in attention deficit hyperactivity disorder adults. A [fluorine-18] fluorodopa positron emission tomographic study. J Neurosci 18:5901-5907.

Eslinger PJ, Damasio AR (1985) Severe disturbance of higher cognition after bilateral frontal lobe ablation: patient EVR. Neurology 35:1731-1741.

Fang J, Yu PH (1994) Effect of L-deprenyl, its structural analogues and some monoamine oxidase inhibitors on dopamine uptake. Neuropharmacology 33:763-768

Garris PA, Wightman RM (1994) Different kinetics govern dopaminergic transmission in the amygdala, prefrontal cortex, and striatum: an in vivo voltammetric study. J Neurosci 14:442-450.

Garris PA, Collins LB, Jones S, Wightman RM (1993) Evoked extracellular dopamine in vivo in the medial prefrontal cortex. J Neurochem 61:637-647.

Goeders NE, Smith JE (1983) Cortical dopaminergic involvement in cocaine reinforcement. Science 221:773-775.

Gorboulev V, Ulzheimer JC, Akhoundova A, Ulzheimer-Teuber I, Karbach U, Quester S, Baumann C, Lang F, Busch AE, Koepsell H (1997) Cloning and characterization of two human polyspecific organic cation transporters. DNA Cell Biol 16:871-881.

Grundemann D, Liebich G, Kiefer N, Koster S, Schomig E (1999) Selective substrates for non-neuronal monoamine transporters. Mol Pharmacol 56:1-10.

Hadfield MG, Nugent EA (1983) Cocaine: comparative effect on dopamine uptake in extrapyramidal and limbic systems. Biochem Pharmacol 32:744-746.

Heikkila RE, Manzino L (1984) Behavioral properties of GBR 12909 and GBR 13098: specific inhibitors of dopamine uptake. Eur J Pharmacol 103:241-248.

Hovevey-Sion D, Kopin IJ, Stull RW, Goldstein DS (1989) Effects of monoamine oxidase inhibitors on levels of catechols and homovanillic acid in striatum and plasma. Neuropharmacology 28:791-797.

Isaac WL, Nonneman AJ, Neisewander J, Landers T, Bardo MT (1989) Prefrontal cortex lesions differentially disrupt cocaine-reinforced conditioned place preference but not conditioned taste aversion. Behav Neurosci 103:345-355.

Izenwasser S, Werling LL, Cox BM (1990) Comparison of the effects of cocaine and other inhibitors of dopamine uptake in rat striatum, nucleus accumbens, olfactory tubercle, and medial prefrontal cortex., Brain Res 520:303-309.

Jentsch JD, Redmond DE, Elsworth JD, Taylor JR, Youngren KD, Roth RH (1997a) Enduring cognitive deficits and cortical dopamine dysfunction in monkeys after long-term administration of phencyclidine. Science 277:953-955.

Jentsch JD, Tran A, Le D, Youngren KD, Roth RH (1997b) Subchronic phencyclidine administration reduces mesoprefrontal dopamine utilization and impairs prefrontal cortical-dependent cognition in the rat. Neuropsychopharmacology 17:92-99.

Juckel G, Mendlin A, Jacobs BL (1999) Electrical stimulation of rat medial prefrontal cortex enhances forebrain serotonin output: implications for electroconvulsive therapy and transcranial magnetic stimulation in depression. Neuropsychopharmacology 21:391-398.

Kalivas PW, Duffy P, DuMars LA, Skinner C (1988) Behavioral and neurochemical effects of acute and daily cocaine administration in rats. J Pharmacol Exp Ther 245:485-492.

Karoum F, Chuang LW, Eisler J, Calne DB, Liebowitz MR, Quitkin FM, Klein DF, Wyatt RJ (1982) Metabolism of (-)deprenyl to amphetamine and methamphetamine may be responsible for (-)deprenyl's therapeutic benefit: a biochemical assessment. Neurology 32:503-509.

Kato T, Dong B, Ishii K, Kinemuchi H (1986) Brain dialysis: in vivo metabolism of dopamine and serotonin by monoamine oxidase A but not B in the striatum of unrestrained rats. J Neurochem 46:1277-1282.
Knoll J (1978) The possible mechanisms of action of (-)deprenyl in Parkinson's disease. J Neural Transm 43:177-198.

Knoll J (1992) The pharmacological profile of (-)deprenyl (Selegiline) and its relevance for humans: a personal view. Pharmacol Toxicol 70:317-321.

Lai JCK, Leung TKC, Guest JF, Lim L, Davison AN (1980) The monoamine oxidase inhibitors clorgyline and L-deprenyl also affect the uptake of dopamine, noradrenaline and serotonin by rat brain synaptosomal preparations. Biochem Pharmacol 29:2763-2767.

Levant B, Grigoriadis DE, De Souza EB (1993) Monoamine oxidase inhibitors inhibit $\left[{ }^{3} \mathrm{H}\right]$ quinpirole binding in rat striatal membranes. Eur J Pharmacol 246:171-178.

Levant B, Moehlenkamp JD, Morgan KA, Leonard NL, Cheng CC (1996) Modulation of $\left[{ }^{3} \mathrm{H}\right]$ quinpirole binding in brain by monoamine oxidase inhibitors: evidence for a potential novel binding site. J Pharmacol Exp Ther 278:145-153.

McElvain JS, Schenk JO (1992) A multisubstrate mechanism of striatal dopamine uptake and its inhibition by cocaine. Biochem Pharmacol 43:2189-2199.

McGregor A, Roberts DC (1995) Effect of medial prefrontal cortex injections of SCH 23390 on intravenous cocaine self-administration under both a fixed and progressive ratio schedule of reinforcement. Behav Brain Res 67:75-80.

McGregor A, Baker G, Roberts DC (1996) Effect of 6-hydroxydopamine lesions of the medial prefrontal cortex on intravenous cocaine selfadministration under a progressive ratio schedule of reinforcement. Pharmacol Biochem Behav 53:5-9.

Meiergerd SM, Schenk JO (1994) Kinetic evaluation of the commonality between the site(s) of action of cocaine and some other structurally similar and dissimilar inhibitors of the striatal transporter for dopamine. J Neurochem 63:1683-1692.

Meiergerd SM, Schenk JO (1995) Measurement of time-resolved kinetics of biogenic amine release and transporter activity by rotating disk electrode voltammetry in vitro. Neuromethod 27. In: Voltammetric methods in brain systems (Boulton AA, Baker G, Adams RN, eds), pp 305-337. Totowa, NJ: Humana.

Meiergerd SM, Patterson TA, Schenk JO (1993) D2 receptors may modulate the function of the striatal transporter for dopamine: kinetic evidence from studies in vitro and in vivo. J Neurochem 61:764-767.

Meiergerd SM, Schenk JO, Sorg BA (1997) Repeated cocaine and stress increase dopamine clearance in rat medial prefrontal cortex. Brain Res 773:203-207.

Merriam EP, Thase ME, Haas GL, Keshavan MS, Sweeney JA (1999) Prefrontal cortical dysfunction in depression determined by Wisconsin card sorting test performance. Am J Psychiatry 156:780-782.

Moghaddam B, Bunney BS (1989) Differential effect of cocaine on extracellular dopamine levels in rat medial prefrontal cortex and nucleus accumbens: comparison to amphetamine. Synapse 4:156-161.

Murphy BL, Arnsten AFT, Goldman-Rakic PS, Roth RH (1996) Increased dopamine turnover in the prefrontal cortex impairs spatial working memory performance in rats and monkeys. Proc Natl Acad Sci USA 93:1325-1329.

Okuda C, Segal DS, Kuczenski R (1992) Deprenyl alters behavior and caudate dopamine through an amphetamine-like action. Pharmacol Biochem Behav 43:1075-1080

Paxinos G, Watson C (1998) The rat brain in stereotaxic coordinates. New York: Academic.

Pehek EA (1999) Comparison of effects of haloperidol administration on amphetamine-stimulated dopamine release in the rat medial prefrontal cortex and dorsal striatum. J Pharmacol Exp Ther 289:14-23.

Piazza PV, Rouge-Pont F, Deminiere JM, Kharoubi M, Le Moal M, Simon H (1991) Dopaminergic activity is reduced in the prefrontal cortex and increased in the nucleus accumbens of rats predisposed to develop amphetamine self-administration. Brain Res 567:169-174.

Povlock SL, Schenk JO (1997) A multisubstrate kinetic mechanism of dopamine transport in the nucleus accumbens and its inhibition by cocaine. J Neurochem 69:1093-1105.

Prasad BM, Hochstatter T, Sorg BA (1999) Expression of cocaine sensitization: regulation by the medial prefrontal cortex. Neuroscience 88:765-774.

Puumala T, Sirvio J (1998) Changes in activities of dopamine and serotonin systems in the frontal cortex underlie poor choice accuracy and impulsivity of rats in an attention task. Neuroscience 83:489-499.

Rajkowska G, Miguel-Hidalgo JJ, Wei J, Dilley G, Pittman SD, Meltzer HY, Overholser JC, Roth BL, Stockmeier CA (1999) Morphometric evidence for neuronal and glial prefrontal cell pathology in major depression. Biol Psychiatry 45:1085-1098.

Schenk S, Horger BA, Peltier R, Shelton K (1991) Supersensitivity to the reinforcing effects of cocaine following 6-hydroxydopamine lesions to the medial prefrontal cortex in rats. Brain Res 543:227-235.

Seamans JK, Floresco SB, Phillips AG (1998) D1 receptor modulation of hippocampal-prefrontal cortical circuits integrating spatial memory with executive functions in the rat. J Neurosci 18:1613-1621.

Sesack SR, Hawrylak VA, Matus C, Guido MA, Levey AI (1998) Do- 
pamine axon varicosities in the prelimbic division of the rat prefrontal cortex exhibit sparse immunoreactivity for the dopamine transporter. J Neurosci 18:2697-2708.

Sharp T, Zetterström T, Ungerstedt U (1986) An in vivo study of dopamine release and metabolism in rat brain regions using intracerebral dialysis. J Neurochem 47:113-122.

Sorg BA, Davidson DL, Kalivas PW, Prasad BM (1997) Repeated daily cocaine alters subsequent cocaine-induced increase in extracellular dopamine in the medial prefrontal cortex. J Pharmacol Exp Ther 281:54-61.

Tanda G, Carboni E, Frau R, DiChiara G (1994) Increase of extracellular dopamine in the prefrontal cortex: a trait of drugs with antidepressant potential? Psychopharmacology 115:285-288.

Tetrud JW, Langston JW (1989) The effect of deprenyl (Selegiline) on the natural history of Parkinson's disease. Science 245:519-522.

Wang XJ (1999) Synaptic basis of cortical persistent activity: the importance of NMDA receptors to working memory. J Neurosci 19:9587-9603.

Weinberger DR (1995) Neurodevelopmental perspectives on schizophrenia. In: Psychopharmacology: the fourth generation of progress (Bloom FE, Kupfer DJ, eds), pp 1171-1183. New York: Raven.

Wheeler DD, Edwards AM, Ondo JG (1993) Dopamine uptake in five structures of the brain: comparison of rate, sodium dependence and sensitivity to cocaine. Neuropharmacology 32:501-508.

Williams GV, Goldman-Rakic PS (1995) Modulation of memory fields by dopamine D1 receptors in prefrontal cortex. Nature 376:572-575.

Wise SP, Murray EA, Gerfen CR (1996) The frontal cortex-basal ganglia system in primates. Crit Rev Neurobiol 10:317-356.

Wolf ME, Dahlin SL, Hu X-T, Xue C-J, White K (1995) Effects of lesions of prefrontal cortex, amygdala, or fornix in behavioral sensitization to amphetamine: comparison with $N$-methyl-D-aspartate antagonists. Neuroscience 69:417-439.

Wu X, Kekuda R, Huang W, Fei Y-J, Leibach FH, Chen J, Conway SJ, Ganapathy V (1998) Identity of the organic cation transporter OCT3 as the extraneuronal monoamine transporter (uptake ${ }_{2}$ ) and evidence for the expression of the transporter in the brain. J Biol Chem 273:32776-32786.

Zahrt J, Taylor JR, Mathew RG, Arnsten AFT (1997) Supranormal stimulation of D1 dopamine receptors in the rodent prefrontal cortex impairs spatial working memory performance. J Neurosci 17:8528-8535.

Zsilla G, Foldi P, Held G, Szekely AM, Knoll J (1986) The effects of repeated doses of (-)deprenyl on the dynamics of monoaminergic transmission. Comparison with clorgyline. Pol J Pharmacol Pharm 38:57-67. 